\title{
Third trimester fetal growth and measures of carbohydrate and lipid metabolism in umbilical venous blood at term
}

John A D Spencer, Tou C Chang, David Crook, Anthony Proudler, Carl V Felton, Stephen C Robson, Martin Hauesler

\begin{abstract}
Aim-To compare measures of carbohydrate and lipid metabolism in umbilical venous blood after birth at term in pregnancies with normal and retarded fetal growth during the third trimester. Methods-Three groups of pregnancies reaching term, in which fetal growth had been prospectively monitored by repeated ultrasound measurements during the third trimester, were studied. Sequential fetal abdominal circumference measurements remained above the 10 th centile in 42 (normal size, normal growth group), below the 10th centile but did not depart further than 1.5 SD (small, normal growth group), or below the 10th centile and subsequently fell away by more than $1.5 \mathrm{SD}$ before delivery (small, growth retarded group). Birthweight, neonatal morphometric measures (ponderal index, mid arm:head circumference ratio, subscapular and triceps skinfold thickness), umbilical venous blood concentrations of glucose, insulin, pro-insulin, des 31,32 proinsulin, total cholesterol, free cholesterol, cholesterol ester, triglycerides, lipoprotein (a), apolipoprotein A-1 and apolipoprotein $B$ were measured.

Results-The median birthweight of the three groups was significantly different (3570, 2569, and $2277 \mathrm{~g}$, respectively). Median values of ponderal index and mid arm:head circumference ratio were significantly lower in the small, growth retarded group and did not differ between the small and normal size groups with normal growth. Both groups with small fetuses had significantly lower mean glucose and cholesterol ester concentrations, and higher mean free cholesterol:cholesterol ester ratios, compared with the normal size, normal growth group. The group showing fetal growth retardation had mean total cholesterol and mean cholesterol ester concentrations that were significantly lower than those of both the other two groups. Mean des 31,32 proinsulin concentrations were low in both groups of small fetuses, but only significantly so in the group without fetal growth retardation. Mean insulin, proinsulin, free cholesterol, triglycerides, lipoprotein(a), apolipoprotein A-1, apolipoprotein $B$ concentrations and the ratio of $A-1: B$ were similar in all three groups.
\end{abstract}

Conclusion-The similarity in the umbilical venous blood carbohydrate and lipid profile at term between pregnancies with documented third trimester fetal growth retardation and those with "genetically" small babies argues against a major role for intrauterine nutritional deprivation as a cause for the association between birthweight and subsequent adult disease. (Arch Dis Child 1997;76:F21-F25)

Keywords: carbohydrate and lipid metabolism; fetal growth retardation; umbilical venous blood; ultrasonography.

A birthweight less than the 10th percentile for gestation is considered important because of associated perinatal morbidity and mortality. ${ }^{1}$ Recent reports have indicated an association between both low birthweight ${ }^{2}$ and small size at birth $^{34}$ with cardiovascular disease and diabetes mellitus in later life. Several studies based on umbilical venous sampling, either before (cordocentesis) or after delivery, have reported significant differences in concentrations of nutritional metabolites between low and normal birthweight for gestational age fetuses. Low birthweight for gestational age fetuses are more often hypoglycaemic, hypoinsulinaemic, hypertriglyceridaemic and hypercholesterolaemic compared with those of normal size..$^{5-10}$

However, not all babies with a low birthweight for gestational age are undergrown because of placental insufficiency and fetal growth retardation. ${ }^{11}$ Abnormal neonatal morphometry may be a better marker than low birthweight to identify babies who are at increased risk of perinatal morbidity. ${ }^{12}{ }^{13} \mathrm{We}$ have shown that fetal growth retardation can be identified before birth when consecutive ultrasound measurements of fetal abdominal circumference fall progressively further from the mean value for gestation age. ${ }^{14}$ As far as we are aware, no study has reported carbohydrate and lipid concentrations in the blood of fetuses with and without prospective ultrasound documentation of fetal growth retardation. As lipoprotein, cholesterol ${ }^{5} 7$ and insulin ${ }^{6}$ have been reported to vary with gestational age at birth, this study was confined to babies who delivered after 36 weeks of gestation.

The aim of this study was to relate blood concentrations of various measures of carbohydrate and lipid metabolism, in the umbilical 
vein after birth at term, to documented fetal growth during the third trimester. Mean plasma glucose, insulin, insulin precursors (proinsulin and des 31,32 proinsulin), cholesterol, triglycerides and apolipoprotein concentrations were compared among three groups: small fetuses with and without evidence of fetal growth retardation, and normally grown fetuses. With one exception, ${ }^{15}$ previous reports of fetal insulin have not considered the influence of insulin precursors which may confound the assessment of insulin status in adults. ${ }^{16}$ To avoid this, we used recently developed assays to obtain separate measures of insulin, proinsulin, and the split proinsulin des 31,32 proinsulin.

\section{Methods}

Women suspected of having a small fetus during the third trimester of pregnancy were recruited for study after referral for an ultrasound scan. In all cases gestational age, assessed by biparietal diameter and femur length at the time of an anomaly scan between 18 and 20 weeks, was within seven days of certain menstrual dates. All delivered after 36 weeks of gestation and none of the mothers received sympathomimetic drugs or steroids. Informed consent was obtained and the study was approved by the hospital ethics committee.

All fetuses were scanned at intervals of one or two weeks on a minimum of three occasions before delivery. Fetal abdominal circumference was measured ${ }^{17}$ on each occasion and expressed as a standard deviation (SD) score (measured value of abdominal circumference minus mean value of abdominal circumference divided by the SD at same gestational age), using mean and SD values derived from our own reference standards. ${ }^{18}$ Growth was assessed as the difference between first and last SD score. A change in SD score of greater than -1.5 was used to define subsequent fetal growth retardation, as we have shown this to be the optimal cutoff in the prediction of abnormal neonatal morphometry indicative of impaired fetal growth. ${ }^{14}$

Umbilical venous blood was obtained by triple clamping of the cord after the birth of the baby and before expulsion of the placenta. A total of $7 \mathrm{ml}$ blood were collected: $4 \mathrm{ml}$ into an tube containing heparin (Vacutainer PST lithium heparin) for assay of immunoreactive insulin, intact proinsulin, and des 31,32 proinsulin; $2 \mathrm{ml}$ into a tube containing heparin (LiHeparin LH/1.3) for lipid and apolipoprotein concentrations; and $1 \mathrm{ml}$ into a sodium fluoride tube for glucose assay. With the exception of the sample for glucose assay, samples were centrifuged within 5 minutes of collection. Aliquots of plasma were stored at $-20^{\circ} \mathrm{C}$ until further analysis.

Glucose concentrations were measured using a glucose oxidase procedure (Yellow Springs Instrument, Ohio, USA) on the same day. Plasma insulin immunoreactivity was measured using a double antibody radioimmunoassay. Intact plasma proinsulin and des 31,32 proinsulin immunoreactivities were measured by immunoradiometric assay using murine monoclonal antibodies. ${ }^{19-20}$ Corrections for intact proinsulin and des 31,32 proinsulin in the insulin radioimmunoassay were performed to minimise confounding by circulating proinsulin. All samples were analysed in a single assay for each analyte. Intra-assay coefficients of variation were $4.4 \%$ for insulin, $4.3 \%$ for intact proinsulin, and $6.3 \%$ for des 31,32 proinsulin. Plasma lipoprotein (a) concentrations were measured using an enzyme linked immunoabsorbent assay (ELISA) kit (Tintelize Lp(a), Biopool, Sweden). Apolipoprotein A-1, apolipoprotein $\mathrm{B}$, and triglyceride concentrations were determined using inhouse immunoturbidimetric assays.

Endogenous free cholesterol was measured using a colorimetric assay supplied by Boehringer Mannheim. Total cholesterol was measured as endogenous free cholesterol plus cholesterol freed by enzymatic cleavage from cholesterol esters. The lipoprotein and cholesterol assays were performed on a discrete automated analyser (Cobas MIRA, Roche Diagnostics, Basel, Switzerland). The intraand interassay coefficients of variations were < $2 \%$ for cholesterol and triglycerides, $<8 \%$ for lipoprotein(a), and $<5 \%$ for apolipoprotein A-1 and apolipoprotein B.

The correlations between different metabolites were assessed using linear regression analysis. As the data for each group were not normally distributed, differences between groups were determined using the MannWhitney $U$ test. Categorical variables were assessed using the $\chi^{2}$ test or Fisher's exact test, where appropriate. Statistical significance was assumed if $\mathrm{P}<0.05$.

\section{Results}

In 49 cases a small for gestational age (SGA) fetus was found (fetal abdominal circumference less than 10th percentile for gestational age) and in 42 cases fetal size was appropriate for gestational age (AGA, fetal abdominal circumference above the 10th percentile for gestational age). ${ }^{16}$ Of the 49 small fetuses, 16 subsequently showed evidence of growth retardation during the third trimester (increments in abdominal circumference measurement fell away from the 10 th centile, such that their final measurement was more than 1.5 SD further from the mean). The remaining 33 small fetuses showed normal growth (increments of abdominal circumference did not deviate more than 1.5 SD from the first value below the 10th centile). The 42 AGA fetuses all had normal ultrasound growth and had normal birthweights. $^{21}$

The median birthweight of the group of growth retarded SGA fetuses was lowest. Demographic and delivery details are shown in table 1. A significantly greater proportion of mothers in the growth retarded SGA group were smokers compared with the other two groups, but there were no differences in incidence of hypertension during pregnancy. There were no significant differences in the median gestational ages at delivery between the groups. Four neonatal morphometric indices of intrauterine nutritional status related well with 
Table 1 Demographic and delivery details according to size and growth

\begin{tabular}{|c|c|c|c|}
\hline & \multicolumn{2}{|c|}{ Small for gestational age (SGA) babies } & \multirow{2}{*}{$\begin{array}{l}\text { Appropriate for } \\
\text { gestational age } \\
(A G A) \text { babies } \\
(n=42)\end{array}$} \\
\hline & $\begin{array}{l}\text { Fetal growth retardation } \\
(n=16)\end{array}$ & Normal growth $(n=33)$ & \\
\hline Maternal age & $24(16-39)$ & $28(17-42)$ & $27(16-39)$ \\
\hline Non-caucasian & $2(13 \%)$ & $2(6 \%)$ & $2(5 \%)$ \\
\hline Primigravidae & $9(56 \%)$ & $20(61 \%)$ & $22(52 \%)$ \\
\hline Smokers & $8(50 \%)^{\star}$ & $5(15 \%)$ & $9(21 \%)$ \\
\hline Hypertension & $2(13 \%)$ & $2(6 \%)$ & $3(7 \%)$ \\
\hline Gestation (days) & $269(253-290)$ & $272(257-292)$ & $274(254-289)$ \\
\hline Duration of labour (hours) & $6(2-12)$ & $6(2-10)$ & $7(2-11)$ \\
\hline Caesarean section for fetal distress & $3(19 \%)$ & $4(12 \%)$ & $3(7 \%)$ \\
\hline Birthweight (g) & $2277(1360-2900)^{\star}+$ & $2569(1701-3120)+$ & $3570(3200-4200)$ \\
\hline Venous $\mathrm{pH}$ & $7.26(7.08-7.40) \dagger$ & $7.29(7.07-7.48) \dagger$ & $7.32(7.22-7.46)$ \\
\hline Venous base deficit & $4.9(0.4-11.8) \dagger$ & $5.1(0.6-10.7) \dagger$ & $3.1(0.3-8.4)$ \\
\hline 5 minute Apgar score $\leq 7$ & $2(13 \%)$ & $4(12 \%)$ & $3(7 \%)$ \\
\hline Admission & $5(31 \%)^{\star}+$ & $1(3 \%)$ & 0 \\
\hline Ponderal index & $2.28(1.90-2.93)^{\star}+$ & $2.45(2.13-3.42)$ & $2.60(2.38-2.98)$ \\
\hline MAC:HC ratio & $0.26(0.23-0.30) \star \dagger$ & $0.28(0.22-0.33)$ & $0.31(0.28-0.35)$ \\
\hline Subscapular skinfold thickness (mm) & $2.7(2.0-4.3)^{\star} \dagger$ & $3.1(2.2-4.4) \dagger$ & $4.6(2.9-6.1)$ \\
\hline Triceps skinfold thickness (mm) & $2.7(1.8-4.2)^{\star} \dagger$ & $3.1(2.2-4.4) \dagger$ & $4.7(3.1-6.2)$ \\
\hline
\end{tabular}

Values are median (range) or numbers (\%).

MAC mid-arm circumference, HC head circumference.

$\star \mathrm{P}<0.05$ vs SGA babies with normal growth.

$+\mathrm{P}<0.05$ vs AGA babies.

Table 2 Metabolic characteristics according to size and growth

\begin{tabular}{|c|c|c|c|}
\hline & \multicolumn{2}{|c|}{ Small for gestational age (SGA) babies } & \multirow{2}{*}{$\begin{array}{l}\text { Appropriate for } \\
\text { gestational age } \\
(A G A) \text { babies } \\
(n=42)\end{array}$} \\
\hline & $\begin{array}{l}\text { Fetal growth retardation } \\
(n=16)\end{array}$ & Normal growth $(n=33)$ & \\
\hline Glucose (mmol/l) & $2.5(1.2-4.8) \dagger$ & $2.7(1.6-5.0) \dagger$ & $3.6(1.8-5.3)$ \\
\hline Insulin $(\mathrm{pmol} / \mathrm{l})$ & $31.2(5.9-272.0)$ & $27.3(2.4-138.7)$ & $37.8(4.1-200.5)$ \\
\hline Proinsulin (pmol/l) & $9.4(4.9-31.4)$ & $8.2(4.2-20.2)$ & $9.3(4.0-23.7)$ \\
\hline Des 31,32 pro-insulin $(\mathrm{pmol} / \mathrm{l})$ & $4.1(1.5-75.1)$ & $4.6(0.9-41.8) \dagger$ & $8.15(0.9-62.1)$ \\
\hline Total cholesterol $(\mathrm{mmol} / \mathrm{l})$ & $1.24(0.74-1.85)^{\star} \dagger$ & $1.38(0.52-2.37)$ & $1.52(1.06-2.58)$ \\
\hline Free cholesterol $(\mathrm{mmol} / \mathrm{l})$ & $0.42(0.29-0.58)$ & $0.45(0.20-0.78)$ & $0.44(0.27-0.78)$ \\
\hline Cholesterol ester $(\mathrm{mmol} / \mathrm{l})$ & $0.84(0.45-1.37)^{\star} \dagger$ & $0.96(0.32-1.64) \dagger$ & $1.08(0.72-1.85)$ \\
\hline Free:cholesterol ester ratio & $0.48(0.35-0.73) \dagger$ & $0.46(0.38-0.64) \dagger$ & $0.40(0.26-0.60)$ \\
\hline Triglycerides $(\mathrm{mmol} / \mathrm{l})$ & $0.44(0.22-1.63)$ & $0.47(0.12-1.43)$ & $0.37(0.13-2.29)$ \\
\hline Lipoprotein (a) (mg/dl) & $0.5(0.5-0.45)$ & $0.5(0.5-10.6)$ & $0.5(0.5-13.9)$ \\
\hline Apolipoprotein A-1 (mg/dl) & $63.0(28.6-72.6)$ & $62.3(30.0-94.3)$ & $68.0(48.5-101.3)$ \\
\hline Apolipoprotein B (mg/dl) & $13.2(6.0-25.0)$ & $15.5(3.8-27.8)$ & $17.3(10.5-30.8)$ \\
\hline $\mathrm{A}-1: \mathrm{B}$ ratio & $4.1(2.2-12.1)$ & $4.4(2.6-23.6)$ & $4.1(2.4-9.1)$ \\
\hline
\end{tabular}

Values are median (range).

$\star \mathrm{P}<0.05$ vs SGA babies with normal growth.

$+\mathrm{P}<0.05$ vs AGA babies.

the prospective grouping by ultrasonography, showing significant differences between the three groups (table 1).

There were no significant correlations between any of the metabolites and gestational age at delivery. There were no significant correlations between plasma glucose concentrations and insulin and insulin precursors in all SGA babies, but a significant correlation between glucose and insulin concentrationa was shown in the group of AGA babies $(\mathrm{r}=0.54, \mathrm{P}=0.001$ ). The median (range) concentrations of lipids and lipoproteins, glucose, insulin and insulin precursors in the three groups are shown in table 2. The group of growth retarded SGA fetuses had significantly lower mean total cholesterol and cholesterol ester compared with the other two groups, but there were no other differences between the two groups of SGA fetuses. Although the mean des 31,32 proinsulin concentration was low in both groups of SGA, the only significant difference was between the SGA group without growth retardation and the AGA group. Both groups of SGA fetuses had significantly lower mean glucose and cholesterol ester, and greater ratios of free cholesterol to cholesterol ester compared with the AGA group. There were no significant differences in mean insulin, proin- sulin, free cholesterol, triglycerides, lipoprotein (a), apolipoprotein A-1, and apolipoprotein $\mathrm{B}$ concentrations or the A-1:B ratio between the three groups.

\section{Discussion}

This is the first study to report metabolic characteristics at term related to third trimester fetal growth patterns. We have already shown that a change in SD score of fetal abdominal circumference measurements during the third trimester not only identifies babies with neonatal morphometric indices indicative of intrauterine nutritional deprivation, ${ }^{14}$ but also predicts suboptimal neonatal outcome. ${ }^{22}$ We have also reported that the mean insulin-like growth factor I concentration was lower in a group of small, growth retarded babies at term compared with groups of small and normal size babies with steady intrauterine growth. ${ }^{23}$ The use of repeated scan measures of abdominal circumference to monitor fetal growth is as effective as the use of repeated estimates of fetal weight based on four fetal morphometric measurements ${ }^{14}$ and is more commonly used in clinical practice. The differences in neonatal morphometric measurements following delivery clearly verify the ability of changes in 
antenatal SD scores, derived from fetal ultrasound measurements of abdominal circumference, to identify fetal growth retardation. The ability to discriminate between SGA fetuses with subsequent growth retardation and SGA fetuses without growth retardation is clinically important as there are differences in perinatal morbidity. ${ }^{1122}$ However, when the SGA group with growth retardation was compared with the SGA group without growth retardation, there were no significant differences in mean glucose, insulin, insulin precursor, free cholesterol, triglycerides and lipoprotein concentrations.

Other workers have shown that small babies are more likely to be hypoglycaemic ${ }^{5} 10$ and hypoinsulinaemic ${ }^{78}$ than normal size babies. The hypothesis that pancreatic cell dysfunction may be involved in the aetiology of hypoinsulinaemia in these babies is supported by necropsy studies of pancreatic tissue in SGA babies $^{24}$ and animal studies. ${ }^{25-26}$ We confirmed that SGA fetuses were significantly hypoglycaemic at birth compared with AGA fetuses, but found no difference between SGA fetuses with a normal rate of intrauterine growth and those with growth retardation. Similarly, we were unable to show significant differences in insulin or proinsulin and des 31,32 proinsulin values, proposed as more refined markers of pancreatic cell dysfunction in adults. ${ }^{16}$ The concentrations of proinsulin and des 31,32 proinsulin observed here in cord blood are higher than those reported in normoglycaemic adults. ${ }^{19}$ Furthermore, insulin propeptides seem to comprise a greater proportion of insulin immunoreactivity, as reported by Hawdon et $a l^{15}$ in neonates. The study design does not allow us to ascribe reasons for the observed higher insulin propeptide values either to alterations in pancreatic function or to changes in plasma clearance mechanisms. The wide range of concentrations, evident in all three groups, could be the result of labour. Insulinlike growth factor binding protein values are higher after delivery following labour compared with elective caesarean section, and are also increased in the presence of fetal distress. ${ }^{27}$ The incidence of caesarean section was significantly different in the three groups in our study, reflecting the higher rate of clinical fetal distress in the small fetuses, especially those with growth retardation. However, the mean duration of the labours in each group was similar. We have previously shown that cortisol concentrations are increased and that thyroid activity is depressed following labour, whereas concentrations of glucose and non-esterified fatty acids remain uninfluenced. ${ }^{28}$

Diaz and coworkers ${ }^{9}$ reported total cholesterol values at birth to be higher in small compared with normal size babies. We found that the mean concentration of total cholesterol was lower in both groups of SGA fetuses, but significantly so in the group with growth retardation. However, this was due primarily to the lower mean values of cholesterol ester in both groups of SGA fetuses, especially the group with growth retardation, resulting in raised mean values of the free cholesterol:cholesterol ester ratio. Diaz et al ${ }^{9}$ showed that this ratio was a marker of lecithin-cholesterol acyltransferase (LCAT) activity, suggesting that, in fetal growth retardation, LCAT deficiency limits the esterification of free cholesterol from high density lipoproteins (HDL) to a cholesterol ester. This would result in raised HDL and triglyceride values. However, we found mean apolipoprotein A-I concentrations, the major protein constituent of HDL and triglycerides, did not significantly differ among the various groups.

Recent reports have indicated associations between low weight or size at birth and subsequent adult diseases such as diabetes mellitus and hypertension. ${ }^{2-4}$ These associations have been attributed to maternal restraint of fetal growth during pregnancy. We wanted to identify a group of term pregnancies complicated by fetal growth retardation in order to compare blood carbohydrate and lipid profiles at birth. The similar lipoprotein, triglyceride, and free cholesterol profiles in all three groups in this study do not provide substantial evidence of a predisposition to atherogenesis as a result of reduced fetal growth. Although our results confirm that there are nutritional consequences of being SGA compared with AGA, major differences were not evident when the groups of SGA fetuses with and without growth retardation were compared. Childhood nutrition has also been shown to have an association with cardiovascular disease in later adult life..$^{29}{ }^{30}$ Therefore, it seems more likely that the epidemiological association found between birthweight and subsequent adult disease reflects familial and genetic tendencies rather than interference with fetal nutrition during the third trimester of pregnancy.

1 Tenovuo AH, Kero PO, Korvenranta HJ. Risk factors associated with severely small for gestational age neonates. $A m$ Perinatol 1988;5:267-71

2 Hales CN, Barker DJP, Clark PMS, Cox LJ, Fall C, Osmond C, Winter PD. Fetal and infant growth and impaired glucose tolerance at age 54. BMF 1991;303:1019-22.

3 Barker DJP, Osmond C, Simmonds SJ, Wield GA. The relation of small head circumference and thinness at birth to death from cardiovascular disease in adult life. $B M F$ 1993;306:422-6.

4 Barker DJP, Martyn CN, Osmond C, Hales CN, Fall CHD. Growth in utero and serum cholesterol concentrations in Growth in utero and serum cholest
adult life. BMF 1993;307:1524-7.

5 Haymond MW, Karl IE, Pagliara AS. Increased gluconeogenic substrate in the small for gestational age infant. $N$ Engl f Med 1974;291:322-8

6 Mestyan J, Soltesz G, Schultz K, Horvath M. Hyperaminoacidaemia due to the accumulation of gluconeogenic amino acid precursors in hypoglycaemic small for gestational age infants. F Pediatr 1975;87:409-14

7 Economides DL, Crook D, Nicolaides KH. Investigation of hypertriglyceridaemia in small for gestational age fetuses. Fetal Ther 1988;3:165-72.

8 Economides DL, Proudler A, Nicolaides KH. Plasma insulin in appropriate- and small-for-gestational age fetuses.

9 Diaz M, Leal C, Cajal R, Jiminez MD, Martinez H, Povoci M, Grande F. Cord blood lipoprotein-cholesterol: Relationship to birth weight and gestational age of newborns. tionship to birth weight and
Metabolism 1989;38:435-8.

10 Hawdon JM, Ward Platt MP, Aynsley-Green A. Patterns of metabolic adaptation for preterm and term infants in the first neonatal week. Arch Dis Child 1992;67:357-65.

11 Patterson RM, Pouliot RN. Neonatal morphometrics and perinatal outcome: Who is growth retarded? Am $\mathcal{F}$ Obstet Gynecol 1987;157:691-3.

12 Villar J, de Onis M, Kestler E, Bolanos F, Cerezo R, Bernedes $\mathrm{H}$. The differential neonatal morbidity of the intrauterine growth retardation syndrome. Am $f$ Obstet Gynecol 1990;163:151-7.

13 Fay RA, Dey PL, Saadie CM, Buhl JA, Gebski VJ. Ponderal index: a better definition of the "at risk" group with intrauterine problems than birthweight-for-gestational age in term infants. Aust NZ 7 Obstet Gynaecol 1991;31:17-9. 
14 Chang TC, Robson SC, Spencer JAD, Gallivan S. Identification of fetal growth retardation: comparison of Doppler waveform indices and serial ultrasound measurements of
abdominal circumference and fetal weight. Obstet Gynecol abdominal circu

15 Hawdon JM, Hubbard M, Hales CN, Clark PMS. Use of specific immunoradiometric assay to determine preterm neonatal insulin-glucose relations. Arch Dis Child 1995;73:F166-9.

16 Temple RC, Carrington CA, Luzio SD, Owens DR, Schneider AE, Sobey WJ, et al. Insulin deficiency in non-insulin dependent diabetes. Lancet 1989;i:293-5.

17 Deter RL, Harrist RB, Hadlock FP, Carpenter RJ. Fetal head and abdominal circumferences: II A critical re-evaluation of the relationship to menstrual age. $f$ Clin Ultrasound 1982;10:365-72.

18 Gallivan S, Robson SC, Chang TC, Vaughan J, Spencer JAD. An investigation of fetal growth using serial
ultrasound data. Ultrasound Obstet Gynecol 1993;3:109-14.

19 Proudler AJ, Godsland IF, Stevenson JC, Insulin propeptides in conditions associated with insulin resistance in
humans and their relevance to insulin measurements. humans and their relevance

20 Sobey WJ, Beer SF, Carrington CA, Clark PMS, Frank BH, Gray IP, et al. Sensitive and specific two-site immunoradiometric assays for human insulin, pro-insulin, 65-66 and 32-33 split pro-insulins. Biochem f 1989;60:534-41.

21 Thomson AM, Billewicz WZ, Hytten FE. The assessment of fetal growth. Br f Obstet Gynaecol 1968;75:903-16.
22 Chang TC, Robson SC, Spencer JAD, Gallivan S. Prediction of perinatal morbidity at term in small fetuses:
comparison of fetal growth and doppler ultrasound. $B r \mathcal{F}$ Obstet Gynaecol 1994;101:422-7.

23 Spencer JAD, Chang TC, Jones J, Robson SC, Preece MA. Third trimester fetal growth and umbilical venous blood concentrations of IGF-1, IGFBP-1 and growth hormone at term. Arch Dis Child 1995;73:F87-90.

24 Van Assche JA, de Prins F, Aerts L, Verians M. The endocrine pancreas in small for dates infants. $B r \mathcal{F}$ Obstet Gynaecol 1977;84:751-3.

25 Fowden AL Comline RS. The effects of pancreatectomy on the sheep fetus in utero. Q $\mathcal{F}$ Exp Physiol 1984;69:319-30. 26 Fowden AL. The role of insulin in prenatal growth. $\mathcal{f} \mathrm{Dev}$ Physiol 1989;12:173-82.

27 Hills FA, Crawford R, Harding S, Farkas A, Chard T. The effects of labor on maternal and fetal levels of insulin-like growth factor binding protein-1. Am 7 Obstet Gynecol 1994; 171:1292-5.

28 Bird JA, Spencer JAD, Mould T, Symonds ME. Endocrine and metabolic adaptation following Caesarean section or vaginal delivery. Arch Dis Child 1996;74:F132-4.

29 Barker DJP, Osmond C. Infant mortality, childhood nutrition, and ischaemic heart disease in England and Wutrition, and ischaemic heart

30 Barker DJP, Winter PD, Osmond C, Margetts B, Simmonds SJ. Weight in infancy and death from ischaemic heart disease. Lancet 1989;ii:577-80 